\title{
Después de la Delegación: la Retracción de la AUTONOMía legislativa del Poder Ejecutivo en Argentina
}

\author{
After Delegation: The Decline of Executive Statutory Discretion \\ in Argentina
}

\section{ERNESTO CALVO}

Universidad de Maryland, EE. UU.

\author{
PAULA CLERICI \\ Universidad Torcuato Di Tella / Universidad de Buenos Aires, Argentina
}

\begin{abstract}
RESUMEN
En este artículo presentamos un nuevo concepto, desacoplamiento ejecutivo, el cual describe una reducción en la capacidad del Poder Ejecutivo para modificar unilateralmente la legislación existente. En este trabajo mostramos que una mayor densidad normativa ha ido limitando a lo largo de décadas la capacidad del Poder Ejecutivo argentino para implementar legislación unilateralmente mediante decretos de necesidad y urgencia. El motivo es que la expansión de la normativa vigente genera una mayor interdependencia entre áreas de legislación, algunas de las cuales están vedadas al Poder Ejecutivo. En la medida en que la red de citación de legislación vigente se expande, esta mayor interdependencia entre áreas de legislación inhibe al Ejecutivo para innovar en áreas que son jurisdiccionalmente contiguas.
\end{abstract}

Palabras clave: Congreso, diputados, Presidente, elecciones, delegación

\begin{abstract}
In this article, we introduce readers to a new concept, Executive Decoupling, which describes a statistical decline in the capacity of the Executive to modify existing legislation by decree. In contrast with Bureaucratic Delegation models, which consider legislative encroachment by the Executive as a contextual event and the result of conflict among the different branches of the government, we show that increased statutory density restrains the ability of the Executive to legislate by decree. The motive is an increase in interdependence between legislative jurisdictions, some of which connect with issues that are restricted to the President. As the citation network of existing legislation expands, increased interdependence between jurisdictions prevents the executive from innovating in contiguous normative issue areas.
\end{abstract}

Keywords: congress, legislators, president, elections, bureaucratic delegation 


\section{INTRODUCCIÓN}

En todas las repúblicas modernas, la normativa vigente es un entramado de leyes, decretos y regulaciones que están interrelacionadas entre sí. En un sistema de división de poderes, esta normativa vigente es el resultado de decisiones políticas, burocráticas y judiciales, realizadas por el Congreso, el poder ejecutivo y los jueces. Este entramado conforma una red de citación con un ADN compartido, en el cual cada actor institucional modifica las decisiones de los otros actores y, de este modo, expresa su poder político.

Este artículo desarrolla el concepto de desacoplamiento ejecutivo para describir el modo en que una mayor vinculación entre normas de distintas áreas de la política pública (mayor densidad normativa), limita la capacidad del Poder Ejecutivo para legislar por decreto ${ }^{1}$. Como discutimos a continuación, cada nueva ley, decreto y acordada judicial, contiene fragmentos de normas anteriores y enlaces a normativas vigentes que describen la contribución de distintos actores institucionales al ADN normativo de un país. Al analizar el proceso de edición de la normativa vigente, ya sea por la aprobación de leyes o de decretos, podemos medir cambios en el balance de poder institucional de los distintos actores.

Conforme las redes normativas de un país se tornan más densas (i.e. mayor interconexión entre áreas legislativas), mayor es la probabilidad de que un proyecto de ley o decreto modifique múltiples áreas simultáneamente (Bonvecchi et al. 2016). Por ejemplo, un proyecto de ley en educación puede requerir la modificación de regulaciones fiscales que afectan la distribución de recursos a las escuelas. Asimismo, un decreto de reordenamiento municipal puede afectar legislación penal o electoral. En países donde existen áreas reservadas sobre las cuales el Ejecutivo no puede legislar por decreto, un incremento de la densidad normativa limita la capacidad del poder de éste de legislar discrecionalmente, dado que mayor interrelación entre las distintas áreas de política pública aumenta la probabilidad de que áreas que están vedadas al Ejecutivo sean afectadas.

Definimos al desacoplamiento ejecutivo [executive decoupling] como la reducción del poder de decreto del Ejecutivo que tiene su origen en una mayor densidad normativa. El concepto guarda relación con la noción de desacoplamiento en biología, física y economía, el cual describe el acto de reducir la contribución estadística que tiene un objeto o mecanismo al interior de un proceso. En nuestro caso, describimos el desacoplamiento ejecutivo como la reducción de la participación estadística del Ejecutivo en la red normativa vigente de un país ${ }^{2}$.

Comenzando por Carey y Shugart (1998), pasando por Negretto (2004), hasta el reciente libro de Palanza (2018), existe una importante literatura que analiza los determinantes estratégicos y contextuales de la implementación de decretos ejecutivos en América Latina. En este artículo contribuimos a esta literatura analizando la relación entre los decretos ejecutivos y las redes de conocimiento legislativo (legislative knowledge networks), las cuales son parte de la familia de estudios sobre "redes de citación" (Bonvecchi et al. 2016; Leich et al. 2007).

2 Vale la pena destacar que nuestra hipótesis difiere radicalmente del modelo de delegación burocrática (Epstein y O'Halloran 1999; Huber y Shipan 2002). En los estudios de delegación burocrática, una mayor 
Para ejemplificar el concepto de desacoplamiento ejecutivo, utilizamos una base de datos que describe las modificaciones realizadas en las leyes y decretos en la Argentina, tal y como han sido recopilados por el Ministerio de Justicia de dicho país. Los resultados empíricos son novedosos y teóricamente importantes, documentando la retracción de la capacidad de legislar por decreto del Ejecutivo en áreas nuevas y también en las complejas.

Este artículo describe tres datos empíricos que han sido pasados por alto en los trabajos sobre democracia delegativa en Argentina. En primer lugar, a lo largo del tiempo, la capacidad de los decretos de necesidad y urgencia (DNU) para innovar en nuevas áreas legislativas ha virtualmente desaparecido ${ }^{3}$. Es decir, actualmente, casi la totalidad de los DNU modifican leyes existentes en lugar de crear nueva política pública. Como contraste, casi un 30 \% de la legislación actual del Congreso no modifica precedentes (leyes existentes), sino que innova en distintas áreas de política pública. En segundo lugar, la legislación reformada por decreto representa menos de un $20 \%$ del total de modificaciones, comparado con un 80 \% que es realizado por el Congreso. Esto se debe a que cada decreto reforma normativa en menos dimensiones o áreas de política pública que aquellas que son modificadas por ley. En tercer lugar, el tiempo que demora un decreto en ser reformado por el Congreso es considerablemente menor que el que toma una ley en ser modificada por un decreto. Visto en forma conjunta: el Ejecutivo en la Argentina no innova en política pública mediante DNU, reforma tan solo una fracción de la legislación existente y, más importante aún, estas modificaciones duran considerablemente menos que aquellas que son implementadas mediante una ley del Congreso. Es decir, a lo largo de décadas, el Congreso argentino ha ido expandiendo su dominio sobre la creación y modificación de la normativa vigente ${ }^{4}$. Más importante aún, mostramos que el desacoplamiento ejecutivo cubre períodos que son arquetípicos del modelo delegativo, como por ejemplo el gobierno de Carlos Menem (19891999) (Ferreira Rubio y Goretti 1996).

complejidad en el diseño de una ley, aumenta la delegación al ejecutivo (Weingast 1984; Laffont y Tirole 1993; Epstein y O’Halloran 1999; Huber y Shipan 2002). Esta complejidad no guarda relación con el entramado legal en el cual se inserta una ley (issue areas) sino con el grado de detalle en el proyecto de ley. Como contraste, nuestro análisis muestra que la mayor densidad normativa en el conjunto de leyes de un país restringe la autoridad del Ejecutivo. No solo la dirección de causalidad difiere, sino que la complejidad se encuentra en la red de citación legislativa antes que en el proyecto de ley.

3 Los DNu en Argentina son un tipo de decreto, quizás el más discrecional de todos, en el que las medidas allí contenidas son de aplicación inmediata. El único requisito que el instrumento debe cumplir es presentar un argumento de las razones que justifican evitar su paso por el órgano legislativo. Sin embargo, los DNU tienen limitaciones constitucionales: no pueden regular materia penal, tributaria, electoral o de régimen de los partidos políticos. Luego son enviados al Congreso para su tratamiento. Así, los DNU corren riesgos políticos en el Parlamento y también legales (Palanza 2018).

4 En trabajos anteriores, Calvo $(2007,2014)$ y junto a Sagarzazu (2011) mostraron que, desde la transición democrática en 1983, el Ejecutivo argentino ha aprobado alrededor de la mitad de los proyectos de ley ingresados al Congreso y, además, gran parte de ellos han sido modificados. Esto ponía en cuestionamiento la idea de un Congreso sin autoridad ni capacidad, a merced de las preferencias del Ejecutivo. En el mismo espíritu, mostramos aquí las limitaciones estructurales que aquejan a la implementación de DNU y explicamos una caída sistemática de su importancia en el entramado legal argentino. 
Para mostrar el desacoplamiento ejecutivo del proceso de creación de leyes, utilizamos la base de datos INFOLEG (s.f.), que incluye todas las leyes nacionales y DNU sancionados en Argentina desde mediados del siglo xIX. Luego de mostrar el proceso histórico de desacoplamiento legislativo, nos enfocamos en el período 1983-2015, modelando la probabilidad de que el Congreso o el Ejecutivo editen la legislación existente mediante nuevas leyes y decretos. Mostramos que una mayor densidad normativa, una mayor complejidad (más precedentes) y una mayor importancia (más citaciones), fortalecen el rol del Congreso.

La organización de este artículo es la siguiente: en la primera sección presentamos nuestro argumento y mostramos cómo la discusión sobre el balance de poderes cambia cuando nos enfocamos en una red normativa antes que en un proyecto de ley o decreto en particular. En la segunda sección presentamos datos descriptivos de las contribuciones relativas del Congreso y el Ejecutivo al entramado legal argentino. En la tercera sección implementamos un modelo multinomial para analizar el proceso de edición de normativa vigente por parte del Congreso y el Ejecutivo. Concluimos —en la cuarta sección- discutiendo algunas extensiones de nuestro trabajo.

\section{REDES DE CITACIÓN LEGISLATIVA Y DESACOPLAMIENTO EJECUTIVO}

En un trabajo reciente, Bonvecchi et al. (2016) mostraban que mientras mayor es el número de normas precedentes que son citadas en una iniciativa legislativa, menor es la probabilidad de que esta se apruebe en el Congreso. La complejidad legislativa limita la probabilidad de sanción de una ley en la medida en que los actores involucrados deben acordar cambios en el statu quo de un mayor número de dimensiones. Complejidad en el proyecto de ley y la fragmentación legislativa — mostraban los autores_-, reducen la probabilidad de sanción de nueva legislación.

El efecto negativo de mayor complejidad en la aprobación de leyes puede ser observado en proyectos del Ejecutivo y del Legislativo, así como también en las distintas áreas legislativas. Dado que en el Congreso no existen áreas o jurisdicciones reservadas, la reducción en la tasa de sanción de leyes afecta tanto a los proyectos de ley propuestos por el Ejecutivo, como también a los formulados por diputados y senadores. No existen, sin embargo, trabajos que muestren el efecto que tiene una mayor complejidad normativa en la aprobación de decretos del Poder Ejecutivo.

En este artículo describimos el resultado que tiene una mayor complejidad normativa en la tasa de aprobación de decretos del Poder Ejecutivo. Mostramos que existen diferencias importantes entre la tasa de aprobación de decretos y de las leyes, conforme la complejidad normativa aumenta. A diferencia de las leyes votadas en el Congreso, en gran cantidad de países los decretos solo pueden ser implementados en algunas áreas de política pública (Bonvecchi y Zelaznik 
2017; Palanza 2018). Por ejemplo, el Poder Ejecutivo argentino no puede constitucionalmente legislar por decreto en temas penales, fiscales o electorales. La existencia de áreas reservadas tiene efectos legislativos distintivos, dado que el aumento en la densidad normativa produce derrames sobre áreas contiguas de política pública.

Conforme aumenta la densidad normativa, decretos introducidos en áreas no reservadas pueden afectar artículos vigentes en legislación que pertenece a espacios que sí lo están. Como resultado, mayor complejidad normativa inhibe la creación en zonas contiguas a las áreas reservadas y, conforme pasa el tiempo, en lugares originalmente separados y distintos. Mayor complejidad normativa, por tanto, produce un desacoplamiento ejecutivo, en la cual el Ejecutivo se ve inhibido de innovar y enfrenta limitaciones importantes en la capacidad de legislar mediante decretos. Estas restricciones no dependen del contexto político ni de un acto delegativo por parte del Congreso. Es decir, una mayor interconexión de la normativa vigente que conecta áreas autorizadas al Ejecutivo con zonas reservadas, limita la capacidad del Ejecutivo para implementar normativa propia. Más aún, este efecto de desacoplamiento ejecutivo [executive decoupling] se ha ido volviendo más pronunciado a lo largo de las últimas décadas.

Podemos pensar intuitivamente en un área reservada como una región en el espacio normativo de la cual el Ejecutivo está vedado. Esta zona, en lugar de permanecer fija a lo largo del tiempo, se expande continuamente en la medida en que distintas áreas de política pública se vinculan entre sí. La interconexión legislativa, en efecto, incrementa el espacio de restricción que está vedado a la discreción presidencial.

El origen del desacoplamiento ejecutivo, por tanto, es la conformación de una red de citación legislativa cada vez más densa, en la cual toda normativa que se propone regular áreas existentes, debe modificar precedentes que intersectan con jurisdicciones reservadas al Congreso. Como en el cuento de Cortázar "Casa tomada" (1951), las áreas en las cuales el Ejecutivo puede innovar se van acotando. En los últimos setenta años, la capacidad de innovación legislativa del Poder Ejecutivo ha ido declinado consistentemente en períodos democráticos.

\section{TRES FENÓMENOS QUE DESCRIBEN DESACOPLAMIENTO EJECUTIVO EN ARGENTINA}

En esta sección presentamos datos estilizados que describen tres fenómenos asociados al desacoplamiento ejecutivo en Argentina: 1) una caída en la creación de "nueva" legislación, legislación que no modifica precedentes, que da cuenta de la mayor densidad de la red legal argentina; 2) una menor supervivencia de los DNU del Ejecutivo; y 3) una menor tasa de modificación de legislación compleja por parte del Ejecutivo. Tomados en conjunto, esto explica que el Ejecutivo innova escasamente, sus DNU sobreviven menos y que la normativa vigente va siendo dominada más extensamente por el Congreso a lo largo del tiempo. 


\section{La retracción de la innovación legislativa mediante decretos}

Describimos, en primer lugar, la caída en el número de DNU que legislan en áreas "nuevas" (áreas en las que no existen precedentes a ser modificados o remplazados). La caída en la tasa de legislación "nueva" muestra que el espacio legislativo se ha ido volviendo más denso, con pocas leyes que en la actualidad pueden aprobarse sin modificar uno o más precedentes. Mientras que a mediados del siglo xx casi un $80 \%$ de las leyes normaban nuevas áreas de política pública, este porcentaje se ha reducido en la actualidad a alrededor de un $10 \%$.

La caída en legislación que no modifica precedentes es considerablemente más pronunciada para el Ejecutivo. Esto se debe a dos motivos: en primer lugar, la gran mayoría de la política pública que es sustantivamente relevante ya tiene normativa que la regula, por lo que la "nueva" legislación a menudo refiere a proyectos de ley que afectan a electorados pequeños o territorialmente concentrados (Bonvecchi et al. 2016). En segundo lugar, el Poder Ejecutivo prefiere mantener control regulatorio sobre áreas en las cuales no existe legislación, evitando lidiar con las ramificaciones legales y políticas de innovar por afuera del Congreso. Los decretos regulativos se apoyan en legislación existente y describe operaciones del Ejecutivo que, si fueran tipificadas por ley, serían sujetas a las restricciones legales de los DNU. Por tanto, donde existe la posibilidad, el Ejecutivo prefiere mantener control sobre la política pública mediante decretos regulatorios, en lugar de utilizar DNU.

Un ejemplo de este tipo de decreto regulatorio es el control - por parte del Ejecutivo- de las retenciones agrícolas, las cuales son establecidas mediante decreto reglamentario por parte de la Secretaría de Comercio. En el marco de la crisis política desatada por la Resolución 125, que propuso un alza en los valores de las retenciones, por ejemplo, se cuestionó si estas retenciones debían ser consideradas como "impuestos", sujetas a ley del Congreso, o como un acto estrictamente administrativo. La decisión de utilizar DNU, por tanto, constriñe la discreción del Ejecutivo, al transformar en materia de ley a políticas que no estarían restringidas si son consideradas materia de regulación. Por tanto, el DNU es utilizado, con cada vez mayor frecuencia, para modificar legislación antes que innovar, tratando de dejar como materia regulatoria todo aquello que no ha sido legislado previamente.

Consideremos que el Poder Ejecutivo puede, en principio, innovar legislativamente mediante: 1 ) proyectos de ley a ser aprobados por el Congreso; 2) DNU o 3) decretos regulatorios que no tienen carácter de ley, pero regulan la actividad del Estado. ¿Cuál debería ser el mecanismo utilizado por el Poder Ejecutivo si le interesa maximizar la autonomía y estabilidad de sus políticas?

Los proyectos de ley aprobados por el Congreso tienen mayor legitimidad y estabilidad en el tiempo, pero pueden ser alterados durante la consideración y sanción por parte de los legisladores, quitándole control sobre la letra chica al 
Ejecutivo y modificando el espíritu que se le quiere dar a la ley. Por tanto, los proyectos de ley tienen mayor legitimidad y estabilidad, pero, a su vez, obligan al Ejecutivo a negociar política pública con los otros actores.

Los decretos administrativos dan la mayor flexibilidad y discreción sobre la implementación de política pública. Sin embargo, estos solo pueden ser implementados en áreas que no son reguladas por ley y que son en su gran mayoría, a su vez, actividades a ser realizadas por agencias estatales.

Los DNU, por otro lado, le permiten al Ejecutivo imponer la política pública que quiere implementar sin tener que negociar con el Congreso y, por tanto, sin tener que sacrificar ninguna cláusula. Sin embargo, estos decretos son legalmente más frágiles, tienen muchas dificultades para legislar en áreas "nuevas" y, como mostraremos a continuación, tienen una mayor probabilidad de ser modificados en un futuro cercano.

La figura 1 describe la proporción de leyes (arriba) o decretos-leyes/DNU (abajo) que no modifican precedentes. Vale la pena destacar que la gran mayoría de estos DNU se encuentran en la línea del cero (modifica precedentes), mientras que los DNU que modifican precedentes casi desaparecen en los últimos veinte años. Entre 1949 y 2017, el espacio legislativo está cada vez más legislado, por lo que disminuye la cantidad de legislación que es "nueva". Eso es cierto tanto de la legislación aprobada por el Congreso, como aquella decretada por el Ejecutivo. Sin embargo, la figura 1 muestra que la legislación "nueva" decretada por el Ejecutivo declina más rápidamente y se encuentra por debajo del 10 \% a partir de la primera década del siglo XXI. 
Figura 1. Proporción de leyes (arriba) y decretos-leyes/DNU (abajo) que no modifican precedentes, 1949-2017
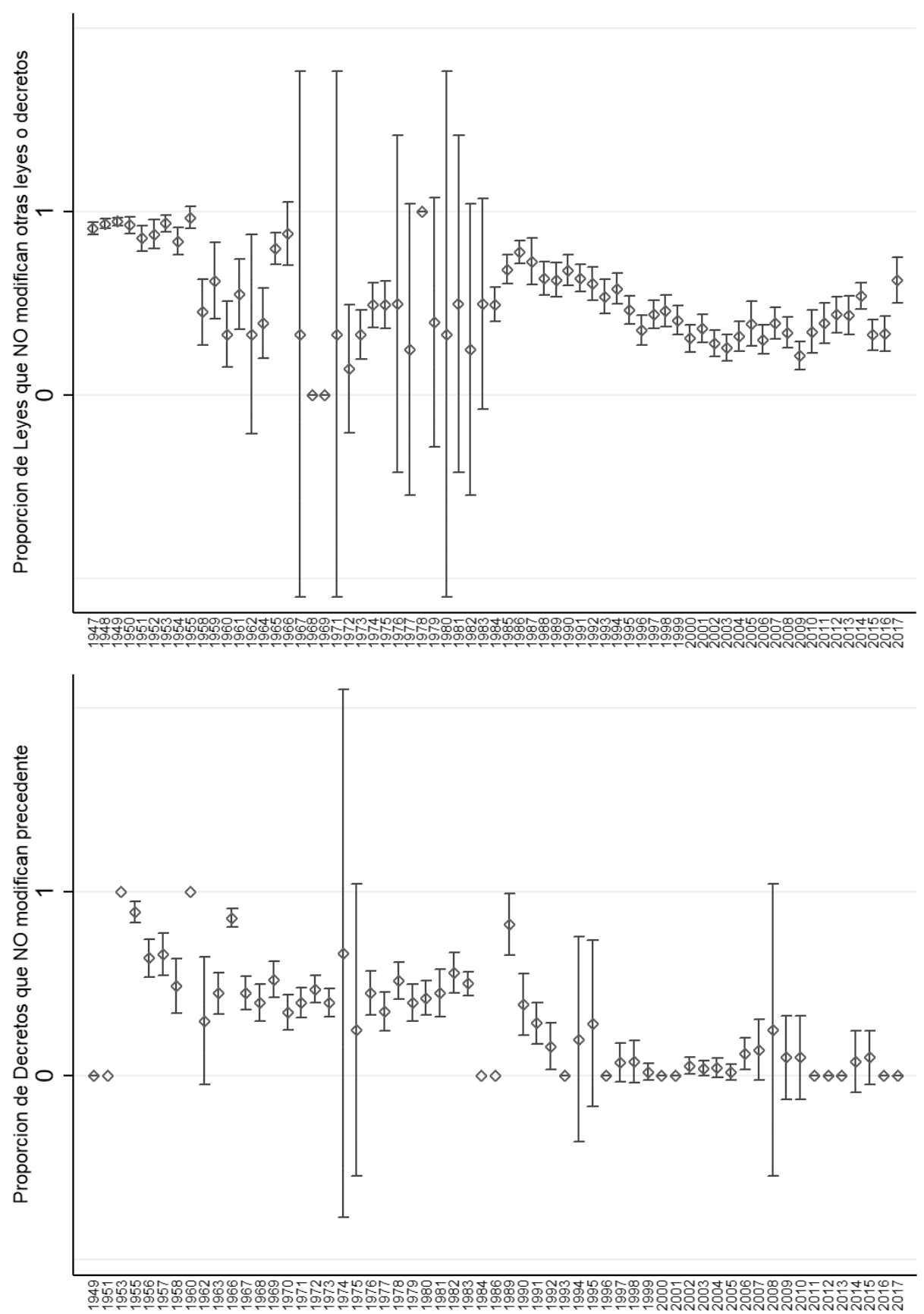

Fuente: elaboración propia con datos de INFOLEG (s.f.)

Nota: proporción de leyes (arriba) o decretos-leyes/DNU (abajo) aprobados y publicados entre 1949 y el 2017. Intervalos de confianza de $95 \%$ 
Entre 1983 y 2017, alrededor de un 40 \% de la legislación aprobada cada año fue original, es decir, no modificó legislación existente. De dichas leyes originales, un $95 \%$ fue aprobada en el Congreso, mientras que tan solo un $5 \%$ fue implementada por DNU (cálculos propios en base a INFOLEG). Cuando estudiamos los DNU que constituyen materia original, la participación del Congreso es aún más dominante.

Pongamos en contexto estos datos para entender qué es lo que definimos como un área no legislada. El 5 de noviembre de 1854, el Congreso argentino sancionó la ley 1, la cual regulaba el crédito fiscal. Dicha ley no modificaba precedentes, como es de esperar, y no sufrió modificaciones. Por otro lado, el 15 de julio de 2010 se aprobó en el Congreso la Ley de Matrimonio Igualitario (ley 26618), la cual habilitaba el casamiento entre ciudadanos del mismo género. Esta ley modificó tres precedentes (el Código Civil de 1869, el Registro Civil de 1969 y el Registro Civil y Capacidad de las Personas de 2008). La figura 1 muestra que, en la actualidad, casi la totalidad de los DNU del Ejecutivo modifican precedentes en su misma área o en áreas normativas contiguas.

\section{El menor tiempo de supervivencia de los DNU}

En la sección anterior mostramos que, a lo largo del tiempo, la reducción en el número de leyes o decretos que innova en nuevas áreas de política pública, ha sido más pronunciada para el Ejecutivo. Esta diferencia, mostraremos más adelante, se debe a que la legislación del Ejecutivo, que no modifica —o que cambia pocos- precedentes, se encuentra en regiones de política pública menos densa, en las que el Poder Ejecutivo prefiere mantener un mayor control mediante decretos regulatorios. El costo de usar decretos regulatorios es que la política pública que prefiere el Gobierno, cambia en el momento en que se deja el cargo. El beneficio, sin embargo, es una mayor discrecionalidad. El problema de la permanencia en el tiempo de las decisiones del Ejecutivo, de hecho, no está tan solo limitada a los decretos administrativos. Como mostramos a continuación, el tiempo de supervivencia de los DNU es también menor que el de las leyes sancionadas por el Congreso.

En la sección anterior mostramos que la Ley de Matrimonio Igualitario modificó simultáneamente tres leyes existentes, una de 1869, otra de 1969 y la última de 2008. El tiempo que transcurre entre el momento que una ley es votada y luego modificada, es lo que definimos como el tiempo de supervivencia. Es decir, cuánto tiempo permanece inalterada una ley del Congreso o DNU. La figura 2 describe la probabilidad de que una ley sancionada por el Congreso o un DNU sobreviva sin modificaciones, cubriendo el período 1983-2016. Para ello, estima un modelo de riesgo proporcional de Cox, donde la variable dependiente es el tiempo transcurrido entre la publicación de una ley o DNU y su modificación por una normativa posterior. 
Figura 2. Tiempo que demoran leyes y DNU para ser modificados: modelo de riesgo proporcional de Cox

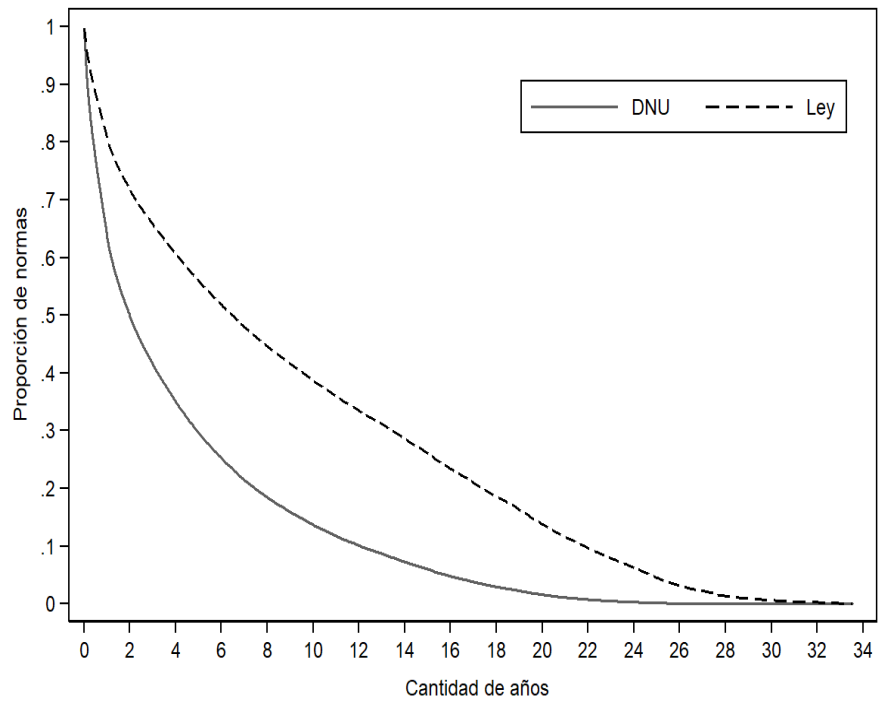

Nota: elaboración propia en base a datos de INFOLEG (s.f.)

Como es de esperar, a medida que pasa el tiempo, disminuye la proporción de normas que permanecen sin modificaciones. Los DNU, sin embargo, sobreviven sin modificaciones un tiempo considerablemente menor que las leyes sancionadas en el Congreso. A los dos años de su publicación, un 38 \% de los DNU emitidos por el Poder Ejecutivo sufrió modificaciones. Como contraste, la proporción de leyes modificadas en los dos años que siguen a su sanción es de tan solo un $20 \%$. Luego de diez años, quedan aún un $40 \%$ de leyes sin modificaciones, sin embargo, tan solo un $15 \%$ de los DNU han evitado ser modificados.

Es decir, la legislación introducida por DNU no solo se ocupa de legislación en áreas ya normadas, como mostramos en la figura 1, sino que, además, tiene una tasa de supervivencia mucho menor que la de las leyes sancionadas por el Congreso.

\section{Desacoplamiento legislativo: datos descriptivos}

Como mostramos en las páginas anteriores, el Congreso argentino es actualmente responsable por casi la totalidad de la legislación que innova en materia de política pública. Los decretos del Ejecutivo, en cambio, se ocupan mayormente de modificar normativa existente. En segundo lugar, mientras que una ley es modificada por un DNU cada 796 días, los DNU son modificados por 
ley cada 244 días. La mayor tasa de remplazo de los DNU limita la contribución del Ejecutivo a la normativa existente que es realizada mediante decretos. Por tanto, el Ejecutivo no innova en áreas de política pública y la supervivencia de sus decretos en el entramado normativo argentino es significativamente menor al de las leyes.

Dado que cada año un 85 \% de la legislación argentina es aprobada por el Congreso, mientras que tan solo un $15 \%$ es implementada mediante DNU, el ADN del Congreso en el total de la legislación argentina es dominante y ha ido ocupando mayores regiones del entramado normativo conforme pasan los años. Es decir, en el largo plazo, la contribución del Congreso al ADN de la legislación argentina ha ido aumentando y la posición del Congreso en la normativa existente se ha ido volviendo cada vez más dominante.

El último dato empírico que destacamos en este artículo es la distinta tasa de modificación de normativa. $\mathrm{O}$, de otro modo, nos ocupamos ahora de la legislación que es modificada por normativa existente. Dado que la legislación que innova en política pública no modifica legislación existente y nace sin modificaciones, la muestra de leyes con la que trabajamos solo puede incluir legislación que ha sufrido alguna modificación. Pensemos que cada ley o DNU puede ser modificado por nuevas leyes o DNU, permitiendo que cada área de política pública tenga distintas contribuciones del Poder Ejecutivo y del Congreso. La figura 3 presenta los datos descriptivos de las modificaciones introducidas por ambos poderes desde la democratización, mostrando el porcentaje de leyes y DNU que son modificados por otras leyes y DNU.

Figura 3. Cantidad de modificaciones a leyes y DNU introducidas por nuevas leyes o decretos en Argentina

\section{3-2015}

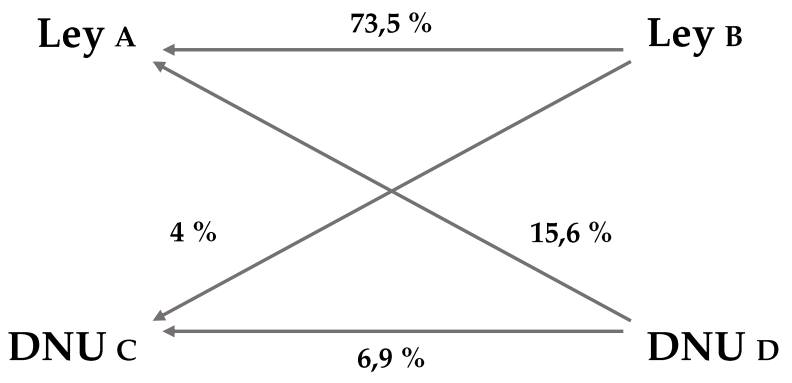

Fuente: elaboración propia con datos de INFOLEG (s.f.). En este modelo analizamos la tasa de modificación de normativa existente por nuevas leyes o decretos. Dado que una ley o decreto que innova en política pública no modifica normativa anterior, y dado que toda nueva ley o decreto nace sin modificaciones, estas opciones no son estimadas en la matriz de probabilidades. 
Como muestra la figura 3, un $77,5 \%$ de las modificaciones a la legislación existente han sido introducidas por ley $(73,5 \%+4 \%)$, en tanto que un $22,5 \%$ de las modificaciones provienen de DNU $(6,9 \%+15,6 \%)$. Vale la pena destacar que la mayor tasa de edición de legislación favorece al Poder Ejecutivo, el cual modifica por DNU en mayor proporción que las reformas de decretos que ocurren mediante leyes del Congreso. Esta mayor tasa de modificación de leyes por parte del Ejecutivo se ha ido acentuando en la medida en que el número de decretos declina como proporción de legislación sancionada por ley. A continuación mostramos que el desacoplamiento legislativo es aún mayor cuando consideramos legislación que es compleja (modifica mayor número de precedentes) e importante (es modificada más frecuentemente).

\section{DESACOPLAMIENTO EJECUTIVO CUANDO LA LEGISLACIÓN ES COMPLEJA E IMPORTANTE}

En las secciones anteriores mostramos que el Poder Ejecutivo innova menos que el Legislativo y que sus DNU sobreviven sin modificaciones un menor tiempo que las leyes sancionadas por el Congreso. También afirmamos que el ADN de la legislación argentina tiene cada vez una menor participación del Poder Ejecutivo, dado que la tasa de modificación observada no logra compensar las diferencias en innovación y la menor supervivencia en el tiempo de los DNU.

Más relevante, sin embargo, es saber cómo se modifica este ADN compartido cuando la legislación a ser considerada es de mayor complejidad e importancia. Es decir, el desacoplamiento del Poder Ejecutivo, ¿es estratégico en la medida en que este se ocupa solo de proyectos importantes? o, por el contrario, ¿es este desacoplamiento más pronunciado en áreas complejas e importantes?

El argumento presentado en las primeras páginas señalaba que una mayor densidad normativa disminuía el peso del Poder Ejecutivo, dado que - esta mayor densidad - aumenta también la probabilidad de que un decreto se vea obligado a modificar artículos vigentes que corresponden a áreas reservadas (i.e. política fiscal, penal o electoral). Dadas las restricciones políticas, constitucionales y legales - argumentamos en la Introducción-, los ejecutivos encuentran dificultades para introducir legislación compleja (legislación que modifica muchas leyes y DNU simultáneamente) e importante (legislación que es relevante para muchas futuras leyes y DNU). Es difícil introducir legislación compleja, argumentamos, porque con mayor frecuencia interactúa con normativa fiscal, electoral o penal que nulifica el DNU. Es también complejo incorporar legislación importante porque el Congreso absorbe rápidamente los cambios del decreto cuando ocurre en áreas densamente legisladas.

Bonvecchi et al. (2016) se refieren a las "redes de conocimiento legislativo" como un entramado complejo de normas donde cada una tiene diferente probabilidad de ser modificada o reemplazada, porque en cada área de política pública son pocas las leyes dominantes. Esto se complejiza a medida que transcurre el 
tiempo, pues la interconexión entre normas tiene un efecto de retroalimentación mutuo: al modificarse una regla, se afecta el funcionamiento de las otras. Esto explica también que, en ciertas áreas de política pública, el Congreso reclame más activamente para sí la sanción de leyes, reclamando para sí mismo espacios que fueron delegados al Ejecutivo (Palanza 2018).

Consideremos, por ejemplo, la figura 4, la cual muestra la cantidad de leyes o decretos que son modificados por cada ley o decreto entre 1983 y 2017. En los últimos treinta años, el promedio de leyes o decretos precedentes que son modificados por cada nueva ley o decreto se triplicó, pasando de alrededor de 0,6 a alrededor de 2 .

Figura 4. Número de precedentes (leyes o decretos) que son modificados por cada nueva ley o decreto, 1983-2017

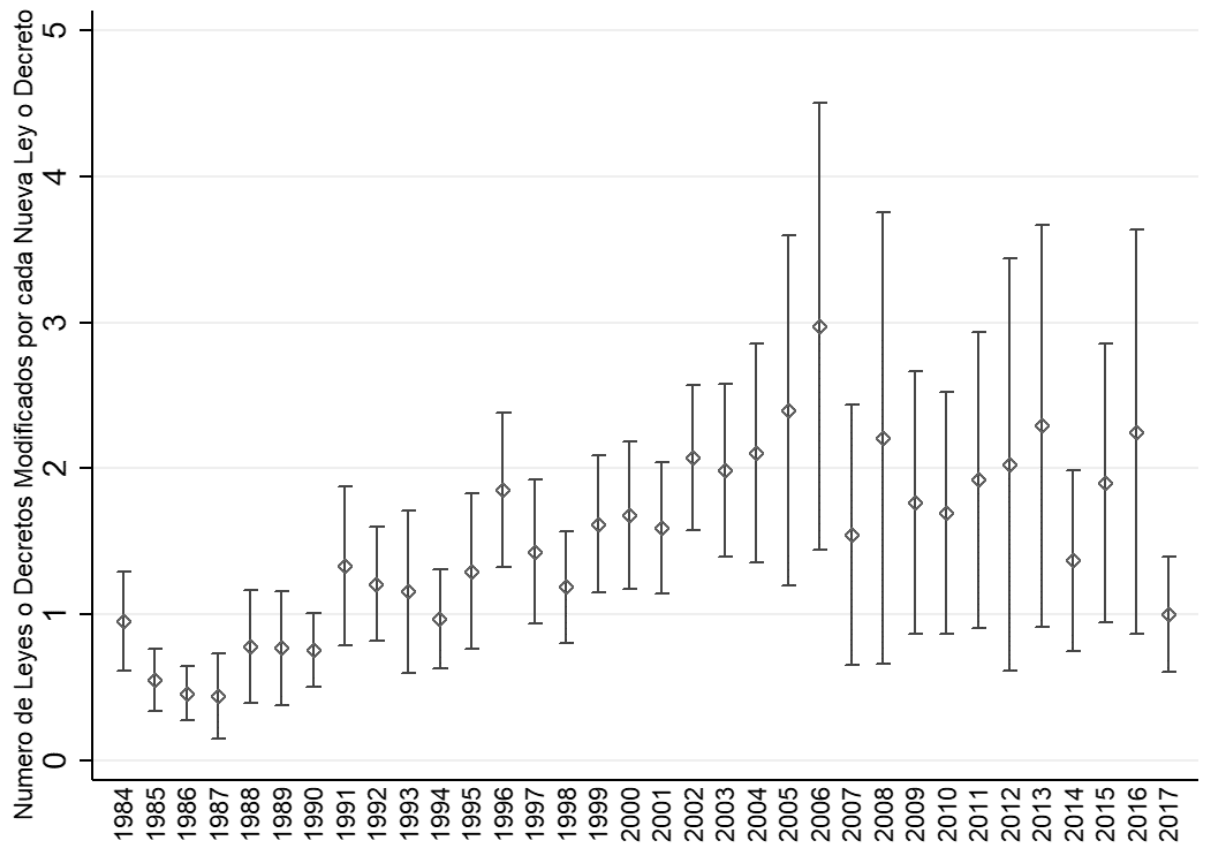

Fuente: elaboración propia en base a datos de INFOLEG (s.f.)

Nota: el número de precedentes modificados incluye todas las leyes y decretos sancionados en el período 19832017, incluyendo aquellos que definimos como "nueva legislación". Es por dicho motivo que el valor puede ser menor a 1 .

\section{Datos y modelos}

Para analizar el efecto de la complejidad e importancia normativa en el balance de poderes entre el Ejecutivo y el Congreso, corremos un modelo multinomial que estima la probabilidad de observar distintos tipos de modificaciones, 
condicional en observar una modificación a una ley o a un DNU por parte de otras leyes o decretos. Es decir, analizamos el efecto de distintos covariados en las probabilidades de modificación de normativa descrita anteriormente, en la figura 3.

La variable dependiente (VD) es uno de cuatro estados, describiendo las cuatro opciones en la figura 3: DNU modifica DNU, ley modifica ley, DNU modifica ley, y ley modifica DNU. Por ejemplo, podemos estimar en qué medida una modificación a legislación que afecta mayor número de precedentes (legislación compleja) puede ser realizada por leyes o por decretos.

Como ya afirmamos, esperamos que mayor complejidad e importancia disminuyan la tasa de modificación de leyes por parte del Ejecutivo. Es decir, esperamos que cuando una norma modifica mayor cantidad de precedentes (mayor complejidad), sea más difícil utilizar DNU y más probable observar leyes del Congreso. Este se debe a que, como mencionamos, mayor complejidad está asociado a una mayor probabilidad de que el proyecto afecte alguna de las áreas que están reservadas para leyes del Congreso.

Asimismo, cuando consideramos normativas de importancia, también esperamos una caída en la tasa de DNU y un aumento en la tasa de leyes, dado que las leyes que son más citadas como precedentes (más modificadas) quedan también contactadas con áreas reservadas en toda legislación futura.

\section{Variables explicativas}

Complejidad: registra las modificaciones realizadas a través del logaritmo natural de la cantidad de veces que una ley o DNU realiza a otras leyes y DNU.

Importancia: mide las modificaciones recibidas mediante el logaritmo natural de la cantidad de veces que un DNU o una ley han sido modificados por otras leyes y DNU.

Tiempo desde la transición democrática: logaritmo de la cantidad de días que la norma permanece inalterada.

Ciclo electoral: variable continua que mide entre 0 y 1 el ciclo del mandato presidencial de cuatro años (o de seis antes de la reforma de la Constitución Nacional en 1994) en su versión lineal y polinómica de tercer grado. Con esta variable, la expectativa es que tanto al comienzo del mandato presidencial, promediando la elección intermedia y al finalizar el ciclo, aumente la probabilidad de que la norma modificatoria sea un DNU, ya sea modificando otro DNU o una ley.

Gobierno dividido: índice de control dividido creado por el proyecto V-Dem. Variable continua en la que el extremo positivo implica que hay partidos diferentes al frente de la Presidencia y de la mayoría en el Congreso. El 
extremo negativo significa que hay control unificado del poder, es decir, el Ejecutivo y la mayoría del Legislativo están controlados por el mismo partido (Coppedge et al. 2018). Calvo (2014) encuentra que el éxito legislativo es mayor cuando ningún bloque alcanza el umbral del $50 \%$ de las bancas. En este sentido, esperaríamos que, cuando el índice que mide el gobierno dividido aumenta, también lo haga la probabilidad de encontrar casos de leyes que modifican otras leyes y DNU.

Fragmentación: considera el número efectivo de bloques en la Cámara Baja. Creemos que, a medida que aumenta la fragmentación, el Ejecutivo recurre a un mayor uso de DNU para compensar las dificultades de apoyo.

Aprobación de la gestión presidencial: variable continúa sistematizada por el proyecto Executive Approval que mide mensualmente el porcentaje de aprobación de la gestión del presidente (Carlin et al. 2016). Esperaríamos que ejecutivos mejor evaluados tengan un mayor espacio para emitir DNU, sea modificando otro DNU o una ley.

Variables dicotómicas por presidencia: el objetivo es analizar qué tan relativamente proclive es cada Ejecutivo a adoptar determinados instrumentos para modificar otros respecto de la Presidencia de Raúl Alfonsín, que es la que se considera como línea de base.

La tabla 1 muestra un modelo multinomial que estima las probabilidades de transición entre los cuatro estados, DNU-DNU, ley-ley (categoría base), DNU-ley y ley-DNu.

Habíamos destacado que la complejidad está de parte del Congreso. En este sentido, cuando el número de leyes y DNU modificados es mayor, es menos probable que la norma modificatoria sea un DNU respecto de la categoría de base. Los coeficientes de las categorías 1 y 4 son negativos y estadísticamente significativos. Contrariamente, la probabilidad de que una ley modifique un DNU (categoría 3) es mayor que la posibilidad de que una ley modifique a otra ley. Estos resultados evidencian que las leyes son más complejas, modifican más normas y es más probable que modifiquen DNU que leyes. 
Tabla 1. Probabilidad de distintos tipos de modificación condicional en observar una modificación

\begin{tabular}{|c|c|c|c|c|}
\hline & $\begin{array}{l}\text { DNU modifica } \\
\text { DNU }\end{array}$ & $\begin{array}{c}\text { Ley modifica } \\
\text { ley (Base) }\end{array}$ & $\begin{array}{c}\text { Ley modifica } \\
\text { DNU }\end{array}$ & $\begin{array}{c}\text { DNU modifica } \\
\text { ley }\end{array}$ \\
\hline Complejidad & $\begin{array}{r}-0,962^{* *} \\
(0,070)\end{array}$ & --- & $\begin{array}{c}0,062^{*} \\
(0,031)\end{array}$ & $\begin{array}{r}-0,532^{* *} \\
(0,048)\end{array}$ \\
\hline Importancia & $\begin{array}{r}-1,113^{* *} \\
(0,054)\end{array}$ & --- & $\begin{array}{r}-0,513^{* *} \\
(0,031)\end{array}$ & $\begin{array}{r}-0,548^{* *} \\
(0,037)\end{array}$ \\
\hline Tiempo desde transición & $\begin{array}{r}-1,196^{* *} \\
(0,334)\end{array}$ & --- & $\begin{array}{r}0,118 \\
(0,149)\end{array}$ & $\begin{array}{r}-1,244^{* *} \\
(0,298)\end{array}$ \\
\hline Ciclo electoral & $\begin{array}{r}0,638 \\
(1,437)\end{array}$ & --- & $\begin{array}{l}-2,497^{*} \\
(1,084)\end{array}$ & $\begin{array}{r}0,671 \\
(1,325)\end{array}$ \\
\hline Ciclo electoral $\wedge^{\wedge}$ & $\begin{array}{l}-2,450 \\
(3,811)\end{array}$ & --- & $\begin{array}{r}10,798^{* *} \\
(2,830)\end{array}$ & $\begin{array}{r}-3,038 \\
(3,480)\end{array}$ \\
\hline Ciclo electoral ${ }^{\wedge} 3$ & $\begin{array}{r}1,196 \\
(2,673)\end{array}$ & --- & $\begin{array}{r}-8,583^{* *} \\
(2,009)\end{array}$ & $\begin{array}{r}1,798 \\
(2,438)\end{array}$ \\
\hline Gobierno dividido & $\begin{array}{l}0,320^{* *} \\
(0,075)\end{array}$ & --- & $\begin{array}{r}0,035 \\
(0,056)\end{array}$ & $\begin{array}{l}0,170^{* *} \\
(0,065)\end{array}$ \\
\hline Fragmentación & $\begin{array}{l}0,949^{* *} \\
(0,195)\end{array}$ & --- & $\begin{array}{r}-0,521^{* *} \\
(0,143)\end{array}$ & $\begin{array}{l}1,120^{* *} \\
(0,149)\end{array}$ \\
\hline Aprobación del PEN & $\begin{array}{l}-0,011^{*} \\
(0,005)\end{array}$ & --- & $\begin{array}{l}0,009^{* *} \\
(0,003)\end{array}$ & $\begin{array}{r}-0,015^{* *} \\
(0,004)\end{array}$ \\
\hline Menem & $\begin{array}{l}5,387^{* *} \\
(1,203)\end{array}$ & & $\begin{array}{l}-0,640^{*} \\
(0,259)\end{array}$ & $\begin{array}{l}5,216^{* *} \\
(1,128)\end{array}$ \\
\hline De la Rúa & $\begin{array}{l}5,547^{* *} \\
(1,334)\end{array}$ & --- & $\begin{array}{l}-0,820^{*} \\
(0,339)\end{array}$ & $\begin{array}{l}5,655^{* *} \\
(1,232)\end{array}$ \\
\hline Duhalde & $\begin{array}{l}7,856^{* *} \\
(1,303)\end{array}$ & --- & $\begin{array}{l}-0,347 \\
(0,327)\end{array}$ & $\begin{array}{l}6,622^{* *} \\
(1,212)\end{array}$ \\
\hline Kirchner & $\begin{array}{l}6,263^{* *} \\
(1,455)\end{array}$ & --- & $\begin{array}{l}-0,938^{*} \\
(0,400)\end{array}$ & $\begin{array}{l}5,734^{* *} \\
(1,340)\end{array}$ \\
\hline Fernández & $\begin{array}{l}3,820^{*} \\
(1,494)\end{array}$ & --- & $\begin{array}{r}-0,532 \\
(0,424)\end{array}$ & $\begin{array}{l}4,084^{* *} \\
(1,365)\end{array}$ \\
\hline Macri & $\begin{array}{l}6,156^{* *} \\
(1,543)\end{array}$ & --- & $\begin{array}{r}-1,445^{* *} \\
(0,456)\end{array}$ & $\begin{array}{l}6,420^{* *} \\
(1,409)\end{array}$ \\
\hline Constante & $\begin{array}{r}3,930 \\
(2,066)\end{array}$ & --- & $\begin{array}{r}1,029 \\
(1,073) \\
\end{array}$ & $\begin{array}{l}2,932^{* *} \\
(1,846)\end{array}$ \\
\hline $\mathrm{N}$ & 5,460 & & 5,460 & 5,460 \\
\hline
\end{tabular}

Fuente: elaboración propia en base a datos de INFOLEG (s.f.)

Nota: ${ }^{*} \mathrm{p}<0,05 ;{ }^{* *} \mathrm{p}<0,01$. Errores estándar entre paréntesis clusterizados por ley modificada. PEN=Poder Ejecutivo nacional

Estas conclusiones se sostienen al analizar la importancia de la norma. En los tres casos que muestra la tabla 1, la probabilidad de ocurrencia es menor a la de la categoría de base. A medida que la norma en cuestión es más veces modificada, es más probable que sea una ley modificando otra ley. La importancia, asimismo, apoya las competencias del Poder Legislativo.

La figura 5 muestra la proporción de DNU y leyes que son reformados por otros DNU y leyes, condicional a la importancia y de la complejidad de la norma modificatoria ${ }^{5}$. Ambos indicadores se miden en base al logaritmo natural de la cantidad de leyes y DNU que la norma modifica (complejidad) o de la cantidad de transformaciones que luego sufre (importancia). 
Habíamos mencionado que es bastante mayor la proporción de casos de leyes que modifican leyes que aquellos de DNU que cambian DNU. Los dos cuadrantes de la izquierda de la figura 5 nos señalan esto fácilmente, al tiempo que indican lo que esperábamos: a medida que la complejidad y la importancia del DNU aumentan, encontramos menor proporción de casos, por lo que la curva es descendente. Sucede lo contrario con las leyes: hay un mayor número que modifican una significativa cantidad de otras normas y que recibirán una importante suma de cambios. Por ejemplo, la ley 26134 es la más compleja del entramado considerado, la cual reforma 138 leyes y DNU (log4.92), fue sancionada en agosto de 2006 con el objetivo de dejar sin efecto el carácter secreto de toda ley que haya sido aprobada con tal condición y prohíbe que ocurra en el futuro.

Figura 5. Importancia y complejidad según tipo de norma modificada y modificatoria (leyes y DNU)
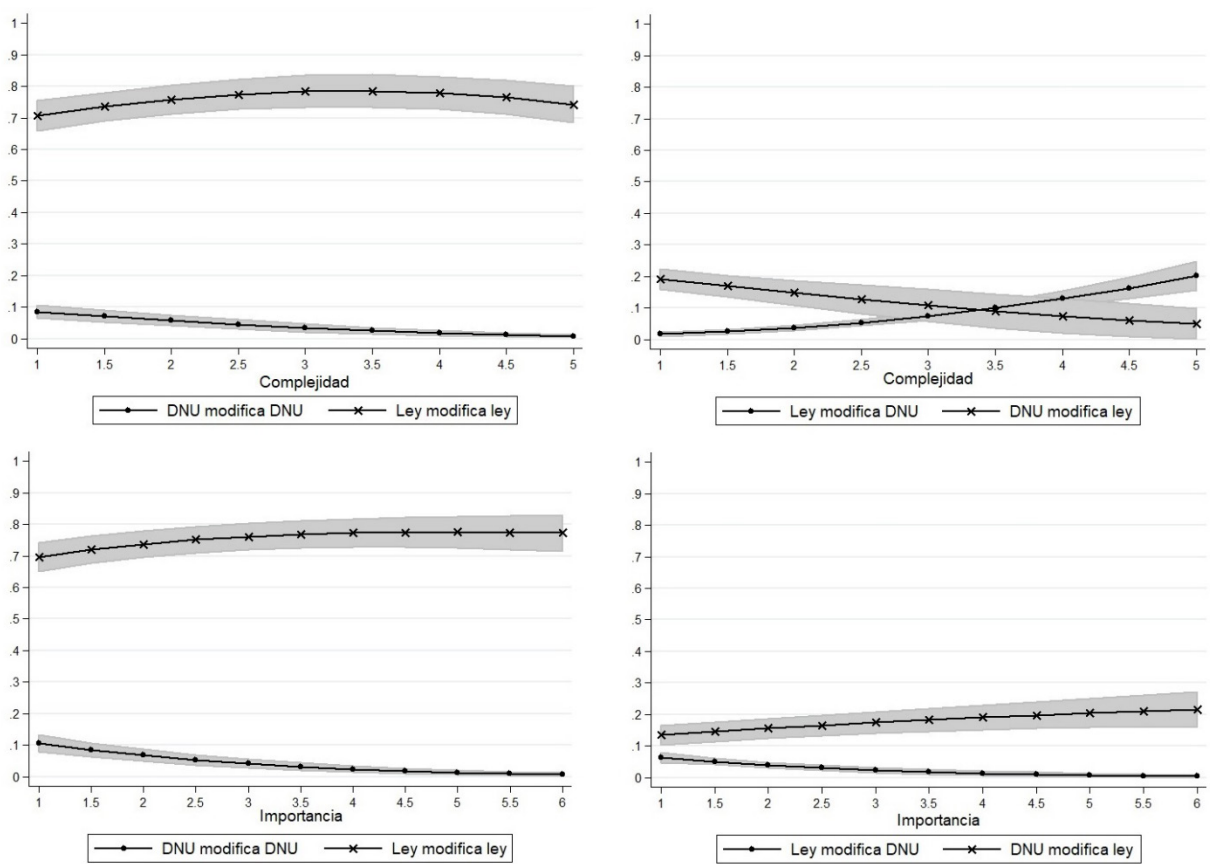

Fuente: tasas de modificación de leyes y DNU condicional a complejidad e importancia (modelos completos en tabla 1). Elaboración propia con datos de INFOLEG (s.f.)

Como la complejidad y la importancia están de parte del Congreso, es esperable que cuando aumenta la cantidad de normas modificadas (complejidad), disminuyen los casos de DNU que reforman leyes, mientras que sucede lo contrario a medida que recibe más trasformaciones (importancia). Cuando el entramado normativo sobre el que se apoya el DNU es más denso, resulta difícil 
que no legisle sobre áreas que lo volverán inconstitucional y, a la vez, cuando es más importante, es más necesaria la vía ordinaria del Congreso.

Ahora bien, los decretos también pueden ser vistos como una forma que tiene el Presidente para forzar al Congreso a que negocie y, a su vez, una manera de mostrarle a la opinión pública que él/ella sigue teniendo control de la iniciativa política. Con frecuencia los ejecutivos diseñan sus decretos con contenidos convergentes con los sancionados por el Congreso, lo que indicaría que buscan evitar conflictos con el Poder Legislativo. De esa forma, este tiene la capacidad de condicionar el uso de poderes unilaterales (Moe y Howell 1999; Bonvecchi y Zelaznik 2017). El Decreto 27/18 de “Desburocratización y simplificación de la administración pública" se ajusta a dicha sospecha. Dos meses luego de emitido el DNU, el Poder Legislativo se encontraba discutiendo su contenido, el que fue desagregado en tres proyectos de ley y, al momento de escribirse estas líneas, ya contaba con la sanción de la Cámara Baja.

\section{Ciclo electoral}

En un sistema presidencial, la cooperación entre los poderes Ejecutivo y Legislativo es una cuestión de necesidad. Aunque, claro está, la noción de cooperación es incluso más imperiosa cuando el Ejecutivo no se encuentra en una situación cómoda en lo que hace al arreglo de bancas. Sin embargo, el ciclo electoral es fundamental, tanto que incluso el margen de maniobra con el que cuenta en el Congreso es independiente de la cantidad de asientos que controla.

Gran número de trabajos en el campo de los estudios legislativos discuten, por un lado, cómo el calendario electoral moldea los horizontes individuales dentro del Congreso y la cohesión partidaria. La cercanía de las elecciones provoca comportamientos diferentes en términos de participación en debates, presentación de proyectos, bloqueos y votaciones (Bräuninger y Debus 2009; Dal Bó y Rossi 2011; Fukumoto y Matsuo 2015; Lucardi y Micozzi 2016). Una noción común que la política comparada sostiene es que cada nuevo gobierno goza de un período de "luna de miel", una especie de tiempo de gracia que la oposición, la sociedad y los medios de comunicación brindan a los recién llegados para empaparse del estado de la cuestión, acomodarse a la gestión, desplegar sus estrategias y poner en marcha la maquinaria.

En este sentido, aunque sabemos que la proporción de leyes es muy superior a la de DNU, al inicio del mandato aumenta su uso. La figura 6 muestra cómo se ve afectada la proporción de casos (eje vertical) de la muestra de leyes y DNU modificándose entre sí, en función del avance del ciclo electoral de cuatro años, lo que dura el período presidencial (el eje horizontal mide el ciclo electoral como un todo entre 0 y 1 ). 
Figura 6. El ciclo electoral de leyes y DNU, 1983-2017
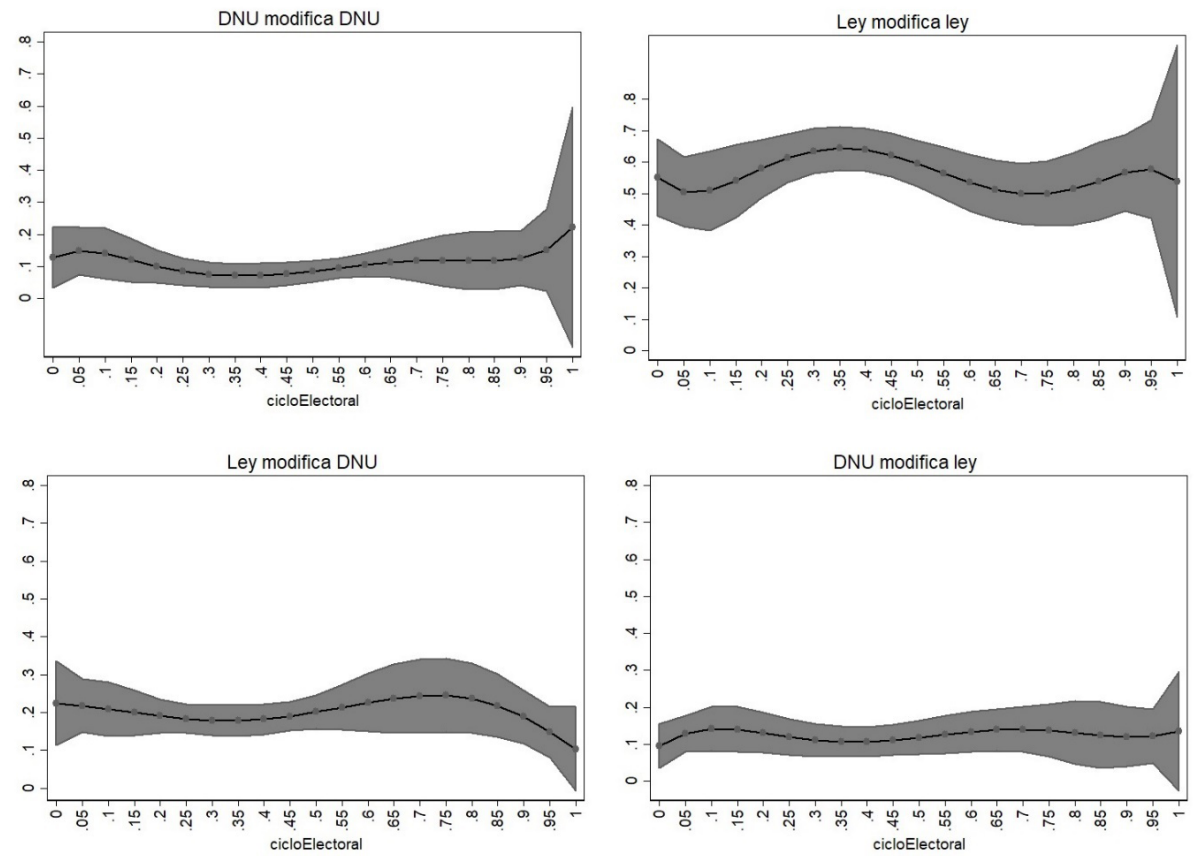

Fuente: elaboración propia con datos de INFOLEG (s.f.)

Es posible observar que durante los primeros cinco meses de comenzado el mandato del Ejecutivo ${ }^{6}$ (0,1 en el eje y), el uso de los DNU aumenta, tanto para modificar otros DNU (gráfico izquierdo superior) como para modificar leyes (izquierdo inferior), aumentando el doble en este último caso. Como destacábamos, al comienzo del mandato el Ejecutivo encuentra un margen más amplio para tomar medidas de manera unilateral, lo cual realiza mediante decretos que cambian elementos de normas anteriores. Por ejemplo, a las dos semanas de asumida la presidencia, Mauricio Macri sancionó el DNU 257/2015 que modifica algunos artículos de cinco leyes nacionales, relacionados con temas como la entrada en vigor del nuevo código procesal penal, un cronograma de implementación progresiva y la asignación de recursos para el Ministerio Público Fiscal.

Al perfilarse la elección legislativa de mitad de período, la actividad del Congreso disminuye, lo que es especialmente notorio en el cuadrante superior derecho que muestra la proporción de leyes que modifican leyes. Los partidos se abocan al armado de las listas y la campaña electoral, mientras que el

6 En el caso de los mandatos de seis años (hasta la reforma de la Constitución Nacional de 1994), esta medida refiere a los primeros nueve meses. Son los casos de las presidencias de Raúl Alfonsín (1983-1989) y la primera de Carlos Menem (1989-1995). 
Congreso ralentiza su actividad. Luego de la elección y la asunción de la nueva Cámara, donde es probable que haya reconfiguración de fuerzas, en el tercer año de gobierno ya se despliegan las decisiones estratégicas para vislumbrar la elección (o reelección) del Ejecutivo. Sigue a la baja la producción de leyes. Estos son dos momentos donde el Ejecutivo toma de nuevo una pequeña ventaja relativa y aumenta la sanción de DNU (es relativa porque la proporción de leyes sigue siendo mayor). Lo mismo al finalizar el ciclo, en los últimos cinco meses antes del término del mandato ${ }^{7}$, los legisladores están en plena actividad de definir sus pases y asunción de nuevas funciones. De la misma manera, cae la producción legislativa y el Ejecutivo apura la utilización de DNU, sea porque se despide del sillón o porque —al cambiar su mandato - también se renueva el Poder Legislativo y las negociaciones cambian de caras.

Cuando hay menor actividad del Congreso, también el Ejecutivo ve constreñida su capacidad de acción. Su agenda legislativa siente de igual forma la demora del trámite parlamentario. Optar por DNU es una vía menor de escape. Pero, como venimos sosteniendo, ni leyes ni DNU implican el imperio de un poder sobre el otro. El entramado normativo se conforma con ADN de ambos actores.

En escenarios de gobierno dividido aumenta la probabilidad de que los presidentes hagan uso de DNU, sea para modificar leyes u otros DNU. Esto sería consistente con la teoría de la delegación, en la cual el Ejecutivo se apoyaría en los DNU como forma de hacer frente a sus débiles poderes partidarios en ambas cámaras (Cox y Morgenstern 2001; Howell 2003; Bonvecchi y Zelaznik 2017). De manera similar, la fragmentación en la Cámara Baja genera el mismo efecto: cuando crece el número de bloques es más probable que, desde la Presidencia, se elija el mecanismo unilateral del decreto para introducir cambios en la legislación. Y cuando se opta por el camino legislativo, sea menos probable que se lo use para cambiar DNU que leyes.

\section{CONCLUSIÓN}

En el largo plazo, el Congreso argentino predomina. Sin importar qué partido ocupe la presidencia, con qué caudal de votos haya ganado, cuál sea el contingente legislativo del partido del Ejecutivo o cuál sea su capacidad para definir la agenda; en el largo plazo las leyes del Congreso dominan el entramado normativo, editando los decretos del Ejecutivo y constriñendo la sanción de nuevos decretos en áreas legislativamente relevantes. Este artículo busca explicar este fenómeno.

El mecanismo que explica la primacía del Congreso en el largo plazo, argumentamos, es relativamente simple: conforme la red normativa argentina se vuelve más densa, una mayor cantidad de legislación modifica precedentes 
en temas que son exclusiva potestad del Congreso. Como resultado, áreas de legislación que en el pasado estaban separadas del resto del entramado legal, comienzan a depender de leyes que solo pueden ser modificadas por el Congreso o que, si fueran reformadas por el Ejecutivo, enfrentarían problemas legales y políticos. Denominamos a este efecto desacoplamiento ejecutivo [executive decoupling], el cual definimos como una expansión de la densidad normativa que conecta un mayor número de áreas legislativas [issue areas] entre sí, limitando los tópicos en los cuales puede innovar el Ejecutivo.

Dadas las restricciones políticas, constitucionales y legales para la utilización de los decretos en temas impositivos, penales y electorales, conforme la red normativa se torna más densa, el presidente encuentra cada vez mayores dificultades para decretar en áreas que son normativamente complejas. Es decir, encuentra dificultades para modificar normativa que simultáneamente afecta a muchas leyes vigentes. Lo mismo ocurre al proponer legislación importante, la cual tiene una alta probabilidad de ser reformada por muchas futuras leyes en áreas que también se encuentran interconectadas. Por tanto, cuando la legislación es compleja o importante, el Congreso eventualmente modifica decretos que intersecta con áreas restringidas.

\section{REFERENCIAS}

Bonvecchi, Alejandro y Javier Zelaznik. 2017. "Poder de decreto presidencial y comportamiento legislativo en Argentina". Revista Uruguaya de Ciencia Política 26 (1): 111-130.

Bonvecchi, Alejandro, Ernesto Calvo y Ernesto Stein. 2016. "Legislative Knowledge Networks, Status Quo Complexity, and the Approval of Law Initiatives". Legislative Studies Quarterly 41 (1): 89-117.

Bräuninger, Thomas y Marc Debus. 2009. "Legislative Agenda-Setting in Parliamentary Democracies". European Journal of Political Research 48 (6): 804-839.

Calvo, Ernesto e Iñaki Sagarzazu. 2011. "Legislator Success in Committee: Gatekeeping Authority and the Loss of Majority Control". American Journal of Political Science 55 (1): $1-15$.

Calvo, Ernesto. 2007. "The Responsive Legislatur Public Opinion and Law Making in a Highly Disciplined Legislature". British Journal of Political Science 37 (2): 263-280.

Calvo, Ernesto. 2014. Legislator Success in Fragmented Congresses in Argentina. Plurality Cartels, Minority Presidents, and Lawmaking. Cambridge: Cambridge University Press.

Carey, John y Mathew Shugart. 1998. Executive Decree Authority. Cambridge: Cambridge University Press.

Carlin, Ryan, Jonathan Hartlyn, Timothy Hellwig, Gregory Love, Cecilia Martínez-Gallardo y Matthew Singer. 2016. Executive Approval Database 1.0. Recuperado de http:/ /www. executiveapproval.org/

Coppedge, Michael, John Gerring, Carl Henrik Knutsen, Staffan I. Lindberg, Svend-Erik Skaaning, Jan Teorell, David Altman, Michael Bernhard, M. Steven Fish, Agnes Cornell, Sirianne Dahlum, Haakon Gjerløw, Adam Glynn, Allen Hicken, Joshua Krusell, Anna Lührmann, Kyle L. Marquardt, Kelly McMann, Valeriya Mechkova, Juraj Medzihorsky, Moa Olin, Pamela Paxton, Daniel Pemstein, Josefine Pernes, Johannes von Römer, Brigitte Seim, Rachel Sigman, Jeffrey Staton, Natalia Stepanova, Aksel Sundstr"om, Eitan Tzelgov, Yi-ting Wang, Tore Wig, Steven Wilson y Daniel Ziblatt. 2018. "V-Dem [Country-Year/Country-Date] Dataset v8". Varieties of Democracy (V-Dem) Project.

Cortázar, Julio. 1951. "Casa tomada”. Bestiario. Buenos Aires: Editorial Sudamericana. 
Cox, Gary y Scott Morgenstern. 2001. "Legislaturas reactivas y presidentes proactivos en América Latina". Desarrollo Económico 41 (163): 373-393.

Dal Bó, Ernesto y Martín Rossi. 2011. "Term Lenght and the Effort of Politicians". The Review of Economic Studies 78 (4): 1237-1263.

Epstein, David y Sharyn O'Halloran. 1999. "Divided Government and the Design of Administrative Procedures: A Formal Model and Empirical Test". The Journal of Politics 28 (2): 376-397.

Ferreira Rubio, Delia y Mateo Goretti. 1996. “Cuando el presidente gobierna solo. Menem y los decretos de necesidad y urgencia hasta la reforma constitucional (julio 1989-agosto 1994). Desarrollo Económico 36 (141): 443-474.

Fukumoto, Kentaro y Akitaka Matsuo. 2015. "The Effects of Election Proximity on Participatory Shirking: The Staggered-Term Chamber as a Laboratory". Legislative Studies Quarterly 40 (4): 599-625.

Howell, William. 2003. Power without Persuasion. The Politics of Direct Presidential Action. Princeton: Princeton University Press.

Huber, John y Charles Shipan. 2002. Deliberate Discretion: The Institutional Foundations of Bureaucratic Autonomy. Cambridge: Cambridge University Press.

INFOLEG. s.f. Buscador de Información Legislativa y Documental del Ministerio de Justicia y Derechos Humanos de la Nación (Argentina). Recuperado de http://www.infoleg. gob.ar/

Laffont, Jean-Jacques y Jean Tirole. 1993. A Theory of Incentives in Procurement and Regulation. Cambridge: MIT Press.

Leicht, Elizabeth, Gavin Clarkson, Kerby Shedden y Marc Newman. 2007. "Large-Scale Structure of Time Evolving Citation Networks". Physics of Condensed Matter 59 (1): 75-83.

Lucardi, Adrián y Juan Pablo Micozzi. 2016. "The Effect of the Electoral Calendar on Politicians' Selection into Legislative Cohorts and Legislative Behavior in Argentina (19832007)". Legislative Studies Quarterly 41 (4): 811-840.

Moe, Terry y William Howell. 1999. "Unilateral Action and Presidential Power: A Theory". Presidential Studies Quarterly 29 (4): 850-873.

Negretto, Gabriel. 2004. "Government Capacities and Policy Making by Decree in Latin America: The Cases of Brazil and Argentina". Comparative Political Studies 37 (5): 531-562.

Palanza, Valeria. 2018. Checking Presidetial Power: Executive Decrees and the Legislative Process in New Democracies. Cambridge: Cambridge University Press.

Weingast, Barry. 1984. "The Congressional-Bureaucratic System: A Principal Agent Perspective (with applications to the SEC)". Public Choice 44 (1): 147-191.

Recibido: 5 de septiembre de 2018

Aceptado: 10 de junio de 2019

Ernesto Calvo. Ph.D en ciencia política por Northwestern University. Es profesor de Gobierno y Política en la Universidad de Maryland (EE. UU.). Su trabajo se centra en el estudio comparado de la representación política, sistemas electorales, redes sociales y congresos. Es autor de NonPolicy Politics (Cambridge University Press 2019), Anatomía Política de Twitter en Argentina (Capital Intelectual 2015), Legislator Success in Fragmented Congresses in Argentina (Cambridge University Press 2014) y La nueva política de partidos (Prometeoo 2005). Sus trabajos, los cuales han sido premiados en múltiples ocasiones por la American Political Science Association (APSA), han sido publicados en revistas especializadas de EE. uU., Europa y América Latina, incluyendo el American Journal of Political Science, el Journal of Politics, el British Journal of Political Science, World Politics, Electoral Studies, Legislative Studies Quarterly, Desarrollo Económico (de Argentina), Política y Gobierno (de México) y Dados (de Brasil). Actualmente está trabajando en un libro que analiza la propagación de mensajes políticos en redes sociales. Correo electrónico: ecalvo@umd.edu 
Paula Clerici. Doctora en ciencia política por la Universidad Torcuato Di Tella. Es Profesora de la Universidad Torcuato Di Tella y la Universidad de Buenos Aires en Argentina. Su trabajo se centra en los estudios electorales, las coaliciones políticas, el sistema de partidos, las relaciones ejecutivolegislativas, los estudios legislativos y el federalismo. Presenta regularmente trabajos en congresos de la disciplina y publica en revistas especializadas. Ha obtenido becas de organismos como Chevening, Fulbright y del Consejo Nacional de Investigaciones Científicas y Técnicas (CONICET) de Argentina para realizar estudios de posgrado en el Reino Unido y estancias de investigación postdoctoral en EE. uU. y Alemania. Correo electrónico: paula.clerici@mail.utdt.edu 